\title{
OPERATION OF A THREE CAVITY SECOND HARMONIC COAXIAL GYROKLYSTRON**
}

\author{
M. Castle, I. Yovchev, W. Lawson, B. Hogan, V.L. Granatstein, and M. Reiser, \\ Institute for Plasma Research, University of Maryland, College Park, MD 20742
}

\begin{abstract}
At the University of Maryland, we have been developing gyroklystrons for advanced electron-positron linear collider applications. We have recently achieved over 80 MW of peak power at $8.6 \mathrm{GHz}$ with a three- cavity first harmonic gyroklystron [2]. The interaction was observed between a $470 \mathrm{kV}, 500 \mathrm{~A}$ annular, rotating beam and a sequence of $\mathrm{TE}_{011}$ coaxial cavities. The efficiency was about $32 \%$ and the gain was about $30 \mathrm{~dB}$. We are currently testing a three-cavity tube that is designed to produce over $100 \mathrm{MW}$ of power at $17.14 \mathrm{GHz}$ with an efficiency of $40 \%$. The input cavity is the same $\mathrm{TE}_{011}$ cavity used in the first harmonic experiment, but the buncher and output cavities are $\mathrm{TE}_{021}$ cavities that operate at twice the cyclotron frequency. In this paper we will describe the design of the circuit and detail the hot test results. Finally, we will also describe the designs of tubes that are capable of higher gain and higher reprate operation.
\end{abstract}

\section{INTRODUCTION}

Various types of microwave power sources are currently under serious investigation as accelerants of particle beams in linear colliders. At the University of Maryland, we have been investigating the prospects and potential of using gyro-amplifiers as drivers for particle beams in a linear accelerator. [1] The main thrust of this work has been geared towards development of the gyroklystron. This device uses discrete cavities along a linear path where the beam-field interaction takes place as in a klystron, while utilizing the helically spiralling electron beam profile typically found in small orbit gyrotrons. The beam-field interaction occurs when the cyclotron frequency of the beam is close to the group velocity of the electromagnetic waves. By employing this mechanism, overmoded cavities may be utilized and higher frequencies of operation may be achieved without decreasing tube radius unduly. Indeed, the gyroklystron has shown the potential to achieve much higher powers at high frequencies $(>30 \mathrm{GHz})$ than that of the conventional linear-beam tubes. Past results achieved at Maryland include $32 \mathrm{MW}$ of power at $19.7 \mathrm{GHz}$ with a $28 \%$ efficiency. This circuit employed was used in the second harmonic mode of operation, had cylindrical geometry, and a smooth-walled output cavity.

Two years ago, a new 100 MW test bed was completed and operated. Coaxial geometry was instituted to reduce mode densities. Because of this geometry, the cavities could be made with abrupt radial transitions, which are beneficial for their ease of manufacture and ease of modification. Tight control over resonant frequency and Q values can be obtained. Two fundamental circuits were tested and results culminated in about $80 \mathrm{MW}$ of power at $8.6 \mathrm{GHz}$ for 1.7 $\mu$ sec at $32 \%$ efficiency. [2] Now testing is underway on a second harmonic three-cavity circuit. The results to date will be reported. Analysis of the limitations and problems encountered will be discussed. An account of the theoretical redesign of the output cavity will be given in section 3. Current efforts for this second harmonic experiment will also be described.

Theoretical efforts on designs for tubes to be made in the future are being performed. These tubes will be made to be compatible with a linear accelerator. Slight modifications to the existing second harmonic system that should improve performance and enable high rep rate operation have been designed. Cold testing has been partially completed for these upgrades.

\section{SECOND HARMONIC TUBE 'SH1'}

The first second harmonic tube to be put on the system in an attempt to create a $100 \mathrm{MW}$ of power was called SH1. Testing on this tube has been completed.

Table 1: System Parameters

\begin{tabular}{|l|c|}
\hline Output Frequency $(\mathrm{GHz})$ & 17.110 \\
\hline Input Frequency $(\mathrm{GHz})$ & $\mathrm{TE}_{02}$ \\
\hline Simulated SH1 efficiency & $41 \%$ \\
\hline Pulse Length $(\mu \mathrm{sec})$ & $\sim 1$ \\
\hline Modulator Voltage $(\mathrm{kV})$ & 460 \\
\hline Beam Current $($ Amps $)$ & 500 \\
\hline Ave. Magnetic Field $(\mathrm{kG})$ & 5.3 \\
\hline Input Cavity Q & $68 \pm 7$ \\
\hline Buncher Cavity Q & $390 \pm 10$ \\
\hline Output Cavity Q & $320 \pm 10$ \\
\hline
\end{tabular}




\subsection{Experimental Results}

Tube SH1 was never able to achieve repeatable amplifier performance. Cathode voltages were usually kept in the $400-470 \mathrm{kV}$ range. Currents were variable, but generally were in the 350-500 A range. The tube was generally quite stable and only small bands of parameter space yielded microwaves. Various magnetic fields detuning and slope combinations and drive power frequencies were attempted to produce amplification to no avail.

\subsection{Analysis of Tube Limitations}

The design of this tube was predicated on the ability to achieve up to 800 A from the electron beam. A 'safe' value of $720 \mathrm{~A}$ was chosen as the nominal design point. This was an attempt to balance both the quest for high power and the need for stability. The maximum current attained during the testing was $530 \mathrm{~A}$. Subsequent large signal code analysis determined that the predicted efficiency at this current was approximately $2 \%$. This value was not achieved because of lower alpha values than those simulated (simulation $\alpha=1.5$ ) from pressure problems and low beam currents. To increase current, most likely a new emitter must be installed. This is a likely possibility for the summer.

\section{THEORETICAL REDESIGN}

A redesign of the output cavity for the lower current values was undertaken for expediency. A value of $540 \mathrm{~A}$ was chosen as the nominal value. Instead of increasing the Q, the cavity was increased in length. Designs with 10, 20 and $30 \%$ increases were tried. The $30 \%$ increase was the most efficient and made to be zero drive stable. Previously, the length to wavelength ratio, $L / \lambda$, in the cavity had been 0.98 , with this new design the value was 1.24. Figure 1 indicates the circuit efficiency with changing values of velocity pitch ratio.

A maximum efficiency of $39.2 \%$ is predicted at $\alpha$ $=1.5$. Figure 2 shows the threshold of oscillation in terms of beam currents as a function of the magnetic field. The operating point of $540 \mathrm{amps}$ is indicated by the horizontal line. As indicated, this tube is theoretically zero-drive stable to all modes, which the previous design was not quite, but had relied on some non-linear suppression of the modes due to bunched beam and magnetic field tapering. Both efficiency and stability simulations were carried out assuming that the axial velocity spread was $6.4 \%$.

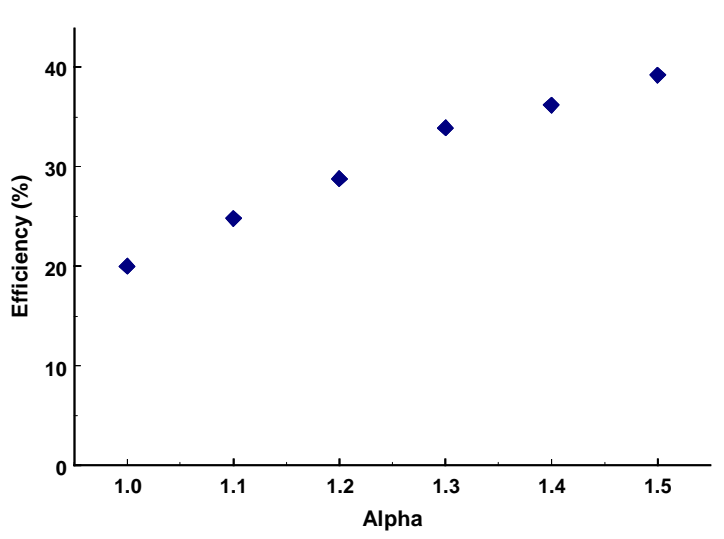

Figure 1: Dependence of efficiency on velocity pitch ratio

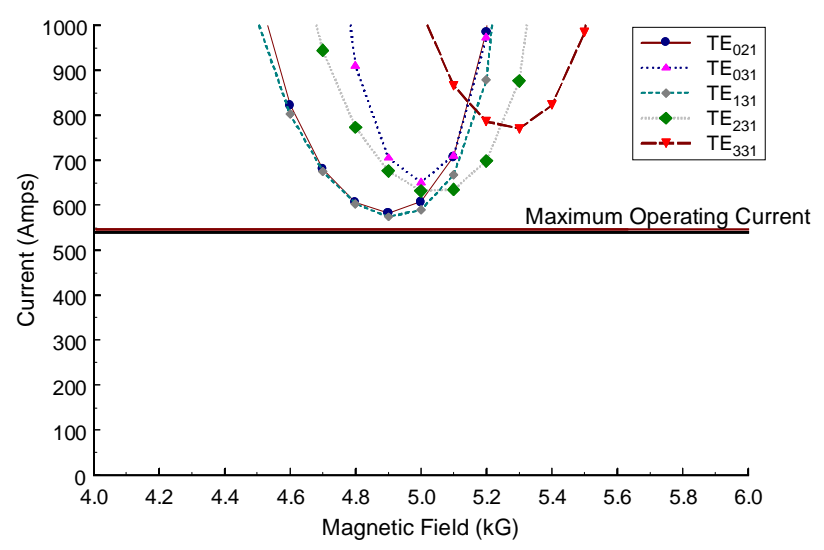

Figure 2: Start oscillation current for main mode competitors

\section{ACCELERATOR COMPATIBLE DESIGNS}

In an effort to make the gyroklystron more compatible with an accelerator, modifications to the system have been started.

\subsection{Dual-Cavity Input Coupler}

In order to more effectively couple input power to the system, a coupler which features two concentric coaxial cavities has been designed using Hewlett-Packard's HFSS. An outer coaxial cavity receives the input signal and resonates in the $\mathrm{TE}_{411}$ mode. Slots on the inner wall of this cavity couple field through to the inner coaxial cavity, which resonates in the $\mathrm{TE}_{011}$ mode. Cold testing 
has verified the design. The resonant frequency and $\mathrm{Q}$ of this inner cavity were measured in the cold test to be $8.570 \mathrm{GHz}$ and 73 .

\subsection{Radial Extracting Output Cavity}

One of the biggest limitations for the present system is the limitation on firing repetition rate. Because this system was mounted horizontally, small tungsten pins are required to hold up the inner conductor of the coaxial circuit. Designs have been completed on HFSS and cold testing is currently underway. The design features the same output cavity as in the second harmonic system except for eight slots cut into the inner conductor wall. These slots couple the power from the $\mathrm{TE}_{021}$ mode in the cavity to the $\mathrm{TE}_{01}$ mode in coaxial waveguide inside. The main benefit is that the inner conductor of the system can be supported from the downstream end because of this extraction method, eliminating the need for the small support pins and freeing up the modulator to increase the repetition rate. In addition, stability may be increased due to the elimination of travelling wave interactions in the post-cavity regions. Finally, conversion from $\mathrm{TE}_{01}$ to other modes is more readily done than from $\mathrm{TE}_{02}$.

\section{CURRENT AND FUTURE WORK}

Cold testing of the redesigned output cavity has been completed. It finalized the dimensions for the output cavity, only a slight change of radius on the inner conductor being necessary to make the resonant frequency $17.11 \mathrm{GHz}$ and the $\mathrm{Q}=320$. The vacuum compatible version of the cavity is now being built and Tube SH2 will be installed and evacuated immediately thereafter. Hot testing of this tube will be complete by summer. Assimilation of the accelerator compatible design will be a focus of the project and should run in parallel with an emitter change in summer. Soon, investigations into the performance of a four-cavity 35 $\mathrm{GHz}$ design will be undertaken.

\section{REFERENCES}

[1] V.L. Granatstein and W. Lawson, "Gyro-Amplifiers as Candidate RF Drivers for TeV Linear Colliders”, IEEE Trans. Plas. Sci., Vol.24, (1996)

[2] W. Lawson, et. al., "High-Power Operation of a Three-Cavity XBand Coaxial Gyroklystron", Phys. Rev. Lett., vol. 81 n.14 (1998) 